\title{
A Direct Meshless Method for Solving Two-Dimensional Second-Order Hyperbolic Telegraph Equations
}

\author{
Fuzhang Wang $\mathbb{D}^{1,2,3}$ and Enran Hou $\mathbb{D}^{3}$ \\ ${ }^{1}$ School of Mathematical and Statistics, Xuzhou University of Technology, Xuzhou 221018, Jiangsu, China \\ ${ }^{2}$ Nanchang Institute of Technology, Nanchang 330044, China \\ ${ }^{3}$ College of Mathematics, Huaibei Normal University, Huaibei 235000, China \\ Correspondence should be addressed to Enran Hou; houenran@163.com
}

Received 9 September 2020; Revised 28 September 2020; Accepted 15 October 2020; Published 10 November 2020

Academic Editor: Imtiaz Ahmad

Copyright (c) 2020 Fuzhang Wang and Enran Hou. This is an open access article distributed under the Creative Commons Attribution License, which permits unrestricted use, distribution, and reproduction in any medium, provided the original work is properly cited.

\begin{abstract}
In this paper, a direct meshless method (DMM), which is based on the radial basis function, is developed to the numerical solution of the two-dimensional second-order hyperbolic telegraph equations. Since these hyperbolic telegraph equations are time dependent, we present two schemes for the basis functions from radial and nonradial aspects. The first scheme is fulfilled by considering time variable as normal space variable to construct an "isotropic" space-time radial basis function. The other scheme considered a realistic relationship between space variable and time variable which is not radial. The time-dependent variable is treated regularly during the whole solution process and the hyperbolic telegraph equations can be solved in a direct way. Numerical experiments performed with the proposed numerical scheme for several two-dimensional second-order hyperbolic telegraph equations are presented with some discussions, which show that the DMM solutions are converging very fast in comparison with the various existing numerical methods.
\end{abstract}

\section{Introduction}

The telegraph equation, which has been used to describe phenomena in various fields, belongs to the hyperbolic partial differential equation scope. For example, the twodimensional second-order hyperbolic telegraph equations can model various physical phenomena in applied sciences and engineering and also has applications in the other fields $[1,2]$. The generalized two-dimensional second-order hyperbolic telegraph equation has the following form:

$$
\mathscr{L} u \equiv \frac{\partial^{2} u}{\partial t^{2}}+2 \alpha \frac{\partial u}{\partial t}+\beta^{2} u-\delta \frac{\partial^{2} u}{\partial x^{2}}-\delta \frac{\partial^{2} u}{\partial y^{2}}=f(x, y, t), \quad t>0
$$

On the physical domain $(x, y) \in \Omega=[0,1] \times[0,1]$, $\alpha>0, \beta$ and $\delta$ are known coefficients, and $f(x, y, t)$ is the source term.

It is well-known that it is difficult to get the analytical solutions for relatively complex problems. Thus, the approximate numerical approximations to the telegraph equation are a better choice. Several numerical methods have been developed and compared to deal with the hyperbolic telegraph equations during the past two decades. In the literature, a three-level implicit unconditionally stable difference scheme for the numerical solution of the hyperbolic equation [3], linear hyperbolic equation with variable coefficients in two space dimensions [4], and multidimensional telegraphic equations [5] are proposed. Bülbül and Sezer [6] proposed a Taylor matrix method for the numerical solution of the two-space dimensional linear hyperbolic equation. A variational iteration method is used to compute the solution for the linear, variable coefficient, fractional derivative, and multispace telegraph equations [7]. The differential quadrature method has been developed for the numerical computation of two-dimensional hyperbolic telegraph equations [8-10]. Hafez [11] proposed a spectral collocation scheme for the numerical solutions of one- and two-dimensional linear telegraph equations with nonlinear forcing term. 
Recently, meshless methods have witnessed the research boom in science and engineering [12-14]. For these branches, Dehghan and his coworkers proposed several meshless methods to investigate the solution of secondorder two-space dimensional linear hyperbolic telegraph equation which include the implicit collocation method [15], the thin plate splines radial basis functions [16], the meshless local Petrov-Galerkin method [17], and the boundary knot method [18]. Recently, the second-order two-space-dimensional telegraph equation in regular and irregular domains are investigated by radial basis function with finite difference scheme [19], the pseudospectral radial basis functions method [20], and the radial basis function method with Crank-Nicolson finite difference scheme [21]. By using the Houbolt method, the solution of the second-order hyperbolic telegraph equation in two space dimensions are solved by the singular boundary method [22] and a hybrid meshless method [23]. Reutskiy et al. [24] proposed a cubic B-spline method based on finite difference and meshless approaches for solving 2D generalized telegraph equations in irregular single and multiconnected domains. These numerical techniques are based on two-level finite difference approximations or integral approximations [25-28].

Based on the abovementioned investigations, we propose a direct meshless method with one-level approximation, which is based on the radial basis functions, for the two-dimensional second-order hyperbolic telegraph equations. Since the hyperbolic telegraph equations are time dependent, we present two schemes for the basis functions from radial and nonradial aspects. The first scheme is fulfilled by considering time variable as normal space variable to construct an "isotropic" space-time radial basis function. The other scheme considered a realistic relationship between space variable and time variable which is not radial. The time-dependent variable is treated regularly during the whole solution process, and the hyperbolic telegraph equations can be solved in a direct way. The rest of this paper is organized as follows. In Section 2, we describe the two-dimensional second-order hyperbolic telegraph equations with initial and boundary conditions. Section 3 introduces the space-time radial and nonradial basis functions. In Section 4, we present the methodology of the direct meshless method (DMM) for the two-dimensional second-order hyperbolic telegraph equations. Several numerical examples are presented to validate the accuracy and stability of the proposed algorithms in Section 5. Some conclusions are given in Section 6 with some additional remarks.

\section{Problem Description}

To seek for the solution of equation (1), one should also consider the initial conditions:

$$
\begin{gathered}
u(x, y, 0)=g_{1}(x, y),(x, y) \in \Omega, \\
u_{t}(x, y, 0)=g_{2}(x, y),(x, y) \in \Omega,
\end{gathered}
$$

and boundary conditions

$$
\mathbb{B} u(x, y, t)=g_{3}(x, y, t),\{(x, y) \mid x=0,1 \text { or } y=0,1\}, \quad t>0,
$$

where $g_{1}(x, y), g_{2}(x, y)$, and $g_{3}(x, y, t)$ are prescribed functions. $\mathbb{B}$ is a boundary operator with $\mathbb{B} u=u$ for Dirichlet boundary and $\mathbb{B} u=(\partial u / \partial x)$ or $\mathbb{B} u=(\partial u / \partial y)$ for Neumann boundary. We aim to seek for the solution of unknown function $u(x, y, t)$.

All meshless numerical techniques for equations (1)-(4) are based on the two-level approximations, most of which are based on the finite difference approximations. In order to overcome the two-level strategy, we propose a direct collocation scheme by using space-time radial and nonradial basis functions.

\section{Formulation of the Space-Time Radial and Nonradial Basis Functions}

It is well known that the radial basis functions (RBFs) are "isotropic" for Euclidean spaces. The most famous Multiquadric (MQ) RBF, which is also named as Kansa's method [29], has the form [30]

$$
\phi_{M Q}\left(r_{j}\right)=\sqrt{1+\left(\varepsilon r_{j}\right)^{2}},
$$

where $r_{j}=\left\|X-X_{j}\right\|=\sqrt{\left(x-x_{j}\right)^{2}+\left(y-y_{j}\right)^{2}}$ and $r_{j}=\| X-$ $X_{j} \|=\sqrt{\left(x-x_{j}\right)^{2}+\left(y-y_{j}\right)^{2}+\left(z-z_{j}\right)^{2}}$ are the Euclidean distance for two-dimensional points $X=(x, y)$ and threedimensional points $X=(x, y, z)$, respectively. $\varepsilon$ is the RBF shape parameter.

For steady-state boundary value problems, the approximate solution can be written as a linear combination of RBFs with $2 \mathrm{D}$ or more higher dimensions. For time-dependent boundary value problems, one should consider the discretization of the time variable in the finite difference way before using the linear combination of RBFs, and this will lead to two-step algorithms.

To obtain a one-step algorithm for the time-dependent problems, we construct a simple direct radial basis function by combining the space variables $x, y$ and time variable $t$ as a point $(x, y, t)$ for the space-time domain $\Omega \times t$. More specifically, the space interval $[0,1]$ is evenly divided into segments firstly $0=x_{0}<x_{1}<\cdots<x_{n}=1$ and $0=y_{0}<y_{1}<$ $\cdots<y_{n}=1$ with corresponding finess $h_{x}=(1 / n)$ and $h_{y}=(1 / n)$. Meanwhile, the time variable is also evenly chosen from the given initial time $t_{0}=0$ to a prescribed final time $t_{n}=T$ by insert some time points $t_{1}, t_{2}, \ldots, t_{n-1}$ with time step $\Delta t=(T / n)$. The corresponding configuration of the space-time coordinate is shown in Figure 1, where "." stands for the value of space coordinate/variables $(x, y)$, “ $*$ " represents the value of time coordinate/variable $t$, and "o" stands for the space-time coordinates $(x, y, t)$.

Similar to the traditional multiquadric radial basis function, the "isotropic" space-time radial basis function can be constructed as 


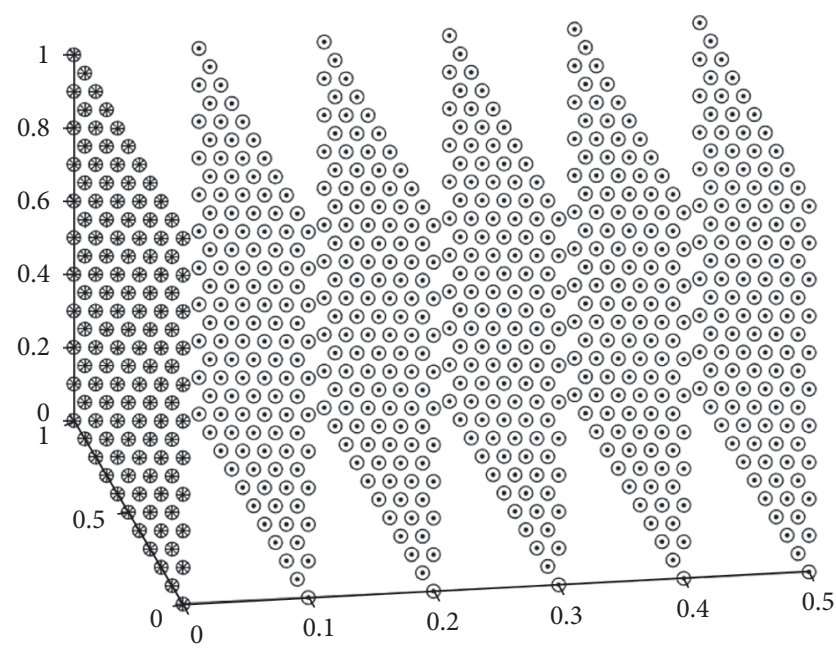

Figure 1: Configuration of the space-time coordinates.

$$
\varphi_{M Q}\left(r_{j}\right)=\sqrt{1+\varepsilon^{2} r_{j}^{2}}
$$

$r_{j}=\left\|P-P_{j}\right\|$ is the Euclidean distance between two space-time coordinates $P=(x, y, t)$ and $P_{j}=\left(x_{j}, y_{j}, t_{j}\right)$. To make a difference between the space variables and time variable, we propose the space-time nonradial basis function which has the following expressions:

$$
\varphi_{N M Q}\left(P, P_{j}\right)=\sqrt{1+\left(x-x_{j}\right)^{2}+\left(y-y_{j}\right)^{2}+\varepsilon^{2}\left(t-t_{j}\right)^{2}}
$$

where $\varepsilon$ can be considered as a parameter which reflects a realistic relationship between space variables $x, y$ and time variable $t$.

Actually, there are several types of definitions of radial or not radial nonmetric space-time radial basis functions with nongeometrical relationship between the space and the time. More details can be found in [31-34].

\section{Implementation of the Direct Meshless Method (DMM)}

4.1. The DMM Solution Procedure. Here, we consider the initial boundary value problem equations (1)-(4) to illustrate the direct meshless method (DMM). Based on the definition of space-time radial or nonradial basis functions, equations (1)-(4) can be solved directly in a one level approximation. The approximate solution of the function $u(x, y, t)$ has the form

$$
\bar{u}(\cdot) \approx \sum_{j=1}^{N} \lambda_{j} \varphi_{j}(\cdot)
$$

We should seek for the unknown coefficients $\left\{\lambda_{j}\right\}_{j=1}^{n}$.

To illustrate the DMM, we choose collocation points on the whole physical domain which include $N_{I}$ internal points $\left\{P_{i}=\left(x_{i}, y_{i}, t_{i}\right)\right\}_{i=1}^{N_{I}}, N_{t}$ initial boundary points $\left\{P_{i}=\left(x_{i}\right.\right.$, $\left.\left.y_{i}, t_{i}\right)\right\}_{i=N_{I}+1}^{N_{I}+N_{t}}$, and $N_{b}$ boundary points $\left\{P_{i}=\left(x_{i}, y_{i}\right.\right.$, $\left.\left.t_{i}\right)\right\}_{i=N_{I}+N_{t}+1}^{N}$. Based on the traditional collocation approach, by substituting equation (8) into equation (1)-(4), we have the following equations:

$$
\begin{aligned}
& \sum_{j=1}^{N} \lambda_{j} \mathscr{L}_{j}\left(P_{i}, P_{j}\right)=f\left(P_{i}\right), \quad i=1, \ldots, N_{I}, \\
& \sum_{j=1}^{N} \lambda_{j} \varphi_{j}\left(P_{i}, P_{j}\right)=g_{1}\left(P_{i}\right), \quad i=N_{I}+1, \ldots, N_{I}+N_{t}, \\
& \sum_{j=1}^{N} \lambda_{j} \frac{\partial \varphi_{j}\left(P_{i}, P_{j}\right)}{\partial t}=g_{2}\left(P_{i}\right), \quad i=N_{I}+N_{t}+1, \ldots, N_{I}+2 N_{t}, \\
& \sum_{j=1}^{N} \lambda_{j} \mathbb{B} \varphi_{j}\left(P_{i}, P_{j}\right)=g_{3}\left(P_{i}\right), \quad i=N_{I}+2 N_{t}+1, \ldots, N,
\end{aligned}
$$

where

$$
\mathscr{L} \varphi_{j}=\frac{\partial^{2} \varphi_{j}}{\partial t^{2}}+2 \alpha \frac{\partial \varphi_{j}}{\partial t}+\beta^{2} \varphi_{j}-\delta \frac{\partial^{2} \varphi_{j}}{\partial x^{2}}-\delta \frac{\partial^{2} \varphi_{j}}{\partial y^{2}} .
$$

It should be noted that the initial boundary points are used twice to cope with initial conditions. Thus, the number of total collocation points $N=N_{I}+2 N_{t}+N_{b}$.

Hence, we should seek for the solution of the following $N \times N$ linear algebraic system:

$$
\mathbf{Q} \Lambda=\mathbf{f},
$$

where

$$
\mathbf{Q}=\left[\begin{array}{llll}
Q_{11} & Q_{12} & Q_{13} & Q_{14} \\
Q_{21} & Q_{22} & Q_{23} & Q_{24} \\
Q_{31} & Q_{32} & Q_{33} & Q_{34} \\
Q_{41} & Q_{42} & Q_{43} & Q_{44}
\end{array}\right],
$$

are $N \times N$ known matrix with submatrices: 
$Q_{11}$ with elements $\mathscr{L} \varphi_{j}\left(P_{i}, P_{j}\right), i, j=1,2, \ldots, N_{I}$

$Q_{12}$ with elements $\mathscr{L} \varphi_{j}\left(P_{i}, P_{j}\right), i=1,2, \ldots, N_{I}, j=$ $N_{I}+1, \ldots, N_{I}+N_{t}$

$Q_{13}$ with elements $\mathscr{L} \varphi_{j}\left(P_{i}, P_{j}\right), i=1,2, \ldots, N_{I}, j=$ $N_{I}+N_{t}+1, \ldots, N_{I}+2 N_{t}$

$Q_{14}$ with elements $\mathscr{L} \varphi_{j}\left(P_{i}, P_{j}\right), i=1,2, \ldots, N_{I}, j=$ $N_{I}+2 N_{t}+1, \ldots, N$

$Q_{21}$ with elements $\varphi_{j}\left(P_{i}, P_{j}\right), i=N_{I}+1, \ldots, N_{I}+N_{t}$, $j=1,2, \ldots, N_{I}$

$Q_{22}$ with elements $\varphi_{j}\left(P_{i}, P_{j}\right), i=N_{I}+1, \ldots, N_{I}+N_{t}$, $j=N_{I}+1, \ldots, N_{I}+N_{t}$

$Q_{23}$ with elements $\varphi_{j}\left(P_{i}, P_{j}\right), i=N_{I}+1, \ldots, N_{I}+N_{t}$, $j=N_{I}+N_{t}+1, \ldots, N_{I}+2 N_{t}$

$Q_{24}$ with elements $\varphi_{j}\left(P_{i}, P_{j}\right), i=N_{I}+1, \ldots, N_{I}+N_{t}$, $j=N_{I}+2 N_{t}+1, \ldots, N$

$Q_{31}$ with elements $\left(\partial \varphi_{j}\left(P_{i}, P_{j}\right) / \partial t\right), i=N_{I}+N_{t}+1$, $\ldots, N_{I}+2 N_{t}, j=1,2, \ldots, N_{I}$

$Q_{32}$ with elements $\left(\partial \varphi_{j}\left(P_{i}, P_{j}\right) / \partial t\right), i=N_{I}+N_{t}+$

$1, \ldots, N_{I}+2 N_{t}, j=N_{I}+1, \ldots, N_{I}+N_{t}$

$Q_{33}$ with elements $\left(\partial \varphi_{j}\left(P_{i}, P_{j}\right) / \partial t\right), i=N_{I}+N_{t}+$

$1, \ldots, N_{I}+2 N_{t}, j=N_{I}+N_{t}+1, \ldots, N_{I}+2 N_{t}$

$Q_{34}$ with elements $\left(\partial \varphi_{j}\left(P_{i}, P_{j}\right) / \partial t\right), i=N_{I}+N_{t}+$ $1, \ldots, N_{I}+2 N_{t}, j=N_{I}+2 N_{t}+1, \ldots, N$

$Q_{41}$ with elements $\mathbb{B} \varphi_{j}\left(P_{i}, P_{j}\right), i=N_{I}+2 N_{t}+1, \ldots$, $N, j=1,2, \ldots, N_{I}$

$Q_{42}$ with elements $\mathbb{B} \varphi_{j}\left(P_{i}, P_{j}\right), i=N_{I}+2 N_{t}+1, \ldots$, $N, j=N_{I}+1, \ldots, N_{I}+N_{t}$

$Q_{43}$ with elements $\mathbb{B} \varphi_{j}\left(P_{i}, P_{j}\right), i=N_{I}+2 N_{t}+1, \ldots$, $N, j=N_{I}+N_{t}+1, \ldots, N_{I}+2 N_{t}$

$Q_{44}$ with elements $\mathbb{B} \varphi_{j}\left(P_{i}, P_{j}\right), i=N_{I}+2 N_{t}+1$, $\ldots, N, j=N_{I}+2 N_{t}+1, \ldots, N$

$$
\Lambda=\left[\begin{array}{l}
\lambda^{1} \\
\lambda^{2} \\
\lambda^{3} \\
\lambda^{4}
\end{array}\right],
$$

is $N \times 1$ vector.

$$
\mathbf{f}=\left[\begin{array}{l}
\mathbf{f} \\
\mathbf{g}_{1} \\
\mathbf{g}_{2} \\
\mathbf{g}_{3}
\end{array}\right],
$$

is $N \times 1$ vector with

$$
\begin{aligned}
f & =\left[f\left(P_{1}\right), f\left(P_{2}\right), \ldots, f\left(P_{N_{I}}\right)\right]^{T}, \\
g_{1} & =\left[g_{1}\left(P_{N_{I}+1}\right), g_{1}\left(P_{N_{I}+2}\right), \ldots, g_{1}\left(P_{N_{1}+N_{t}}\right)\right]^{T}, \\
g_{2} & =\left[g_{2}\left(P_{N_{I}+N_{t}+1}\right), g_{2}\left(P_{N_{I}+N_{t}+2}\right), \ldots, g_{2}\left(P_{N_{1}+2 N_{t}}\right)\right]^{T}, \\
g_{3} & =\left[g_{3}\left(P_{N_{I}+2 N_{t}+1}\right), g_{3}\left(P_{N_{I}+2 N_{t}+2}\right), \ldots, g_{3}\left(P_{N}\right)\right]^{T} .
\end{aligned}
$$

Equation (14) can be solved by the backslash computation in MATLAB codes. From the above procedures, we can find that the implementation of the proposed direct meshless method is very simple. Finally, by substituting the determined coefficients into equation (8), we obtain the approximate solution:

$$
\bar{u}(\cdot) \approx \sum_{j=1}^{N} \lambda_{j} \varphi_{j}(\cdot) .
$$

4.2. Algorithm of the DMM. In this part, we summarize steps of the given method as an implementation algorithm as follows:

Step 1: enter the given functions $f(x, y, t), g_{1}(x, y)$, $g_{2}(x, y)$, and $g_{3}(x, y, t)$, and the real constants $\alpha, \beta$, and $\delta$

Step 2: select the value $n$ which corresponds to the total collocation number $N$

Step 3: find the space-time collocation points $\left(x_{i}, y_{i}, t_{i}\right), I=1,2, \ldots, N$ which include $N_{I}$ internal points $\left\{P_{i}=\left(x_{i}, y_{i}, t_{i}\right)\right\}_{i=1}^{N_{I}}, N_{t}$ initial boundary points $\left\{P_{i}=\left(x_{i}, y_{i}, t_{i}\right)\right\}_{i=N_{I}+1}^{N_{I}+N_{t}}$, and $N_{b}$ boundary points $\left\{P_{i}=\left(x_{i}, y_{i}, t_{i}\right)\right\}_{i=N_{I}+N_{t}+1}^{N}$

Step 4: compute the matrix $\mathbf{Q}$ and the vector $\mathbf{f}$

Step 5: solve the system $\mathbf{Q} \Lambda=\mathbf{f}$

Step 6: substitute the determined coefficients from $\Lambda$ into (9) and construct the approximate solution $\bar{u}(x, y, t)$ in (19)

\section{Numerical Experiments}

In this section, several examples are considered to validate the DMM. For fair comparison with the other numerical methods, we use the maximum absolute error (MAE), relative error $\left(L_{2}\right)$, and root mean square error (RMSE). The RMSE and $L_{2}$ errors are defined as below $[35,36]$ :

$$
\operatorname{RMSE}=\|u(P)-\widetilde{u}(P)\|_{2}=\sqrt{\frac{1}{\mathrm{NT}} \sum_{j=1}^{\mathrm{NT}}\left|u\left(P_{j}\right)-\widetilde{u}\left(P_{j}\right)\right|^{2}}, L_{2}=\|u(P)-\widetilde{u}(P)\|_{2}=\sqrt{\frac{\sum_{j=1}^{\mathrm{NT}}\left|u\left(P_{j}\right)-\widetilde{u}\left(P_{j}\right)\right|^{2}}{\sum_{j=1}^{\mathrm{NT}}\left|u\left(P_{j}\right)\right|^{2}}},
$$


where $u$ is the analytical solution at test points $\left\{P_{j}\right\}_{j=1}^{\mathrm{NT}}$ and $\tilde{u}$ is the numerical solutions at the test points $\left\{P_{j}\right\}_{j=1}^{\mathrm{NT}}$. NT is the number of test points on the physical domain. For the choice of parameter $\varepsilon$ in the DMM, we consider a prior determination. More specifically, the collocation points are fixed to find the quasi-optimal parameter; then, it will be used for the other calculations. The optimal choice of space-time radial or nonradial basis function parameter is similar with the other traditional radial basis functions. For more details about this topic, one can be found in $[37,38]$ and references therein.

We denote the "isotropic" space-time radial basis function method as DMM1 and the space-time nonradial basis function as DMM2 in the following numerical investigations.

5.1. Example 1. First, we consider the hyperbolic telegraph equation (1) with the initial conditions

$$
\begin{aligned}
u(x, y, 0) & =\sin x \sin y, \quad(x, y) \in[0,1] \times[0,1 r], \\
u_{t}(x, y, 0) & =0, \quad(x, y) \in[0,1] \times[0,1],
\end{aligned}
$$

and boundary conditions

$$
\begin{aligned}
& u(0, y, t)=u(x, 0, t)=0, \quad 0 \leq t<1, \\
& u(1, y, t)=\cos (t) \sin (1) \sin (y), \quad 0 \leq t<1 \\
& u(1, y, t)=\cos (t) \sin (1) \sin (y), \quad 0 \leq t<1 .
\end{aligned}
$$

The corresponding coefficients are $\alpha=1, \beta=1$, and $\delta=$ 1 with analytical/exact solution:

$$
u(x, y, t)=\cos (t) \sin (x) \sin (y) .
$$

The source term is

$$
f(x, y, t)=2(\cos (t)-\sin (t)) \sin (x) \sin (y) .
$$

For $h_{x}=h_{y}=\Delta t=(1 / 9)$, the total collocation point number is $N=1036$. The DMM results with root mean square errors (RMSE), $L_{2}$, and maximum errors, $L_{\infty}$, are listed in Table 1 for different times $t=0.5, t=1$, and $t=2$. We also compare our results with the ones reported in $[8-10,19,20]$. It should be pointed that our time step $\Delta t=(1 / 9)$, which will lead to less computations, is larger than the one $\mathrm{d} t=0.01$ in $[8-10]$ and $\mathrm{d} t=0.001$ in $[19,20]$. However, all the root mean square errors (RMSE), $L_{2}$, and maximum errors, $L_{\infty}$, of the two DMM schemes perform better than the other numerical methods. We can see that the results obtained by the proposed method are in excellent agreement with the exact solutions. The maximum absolute errors remain stable around $L_{\infty} \approx 10^{-7}$ for all times tested. Since the two DMM schemes perform similar results, the DMM2 scheme will be eliminated in the rest numerical results.

The absolute error in Figure 2 shows that the results of our DMM method are more satisfactory and reliable.

5.2. Example 2. Consider the hyperbolic telegraph equation (1) with the corresponding initial conditions
TABle 1: Numerical results for Example 1.

\begin{tabular}{lcccc}
\hline Methods & $t$ & $L_{2}$ & RMSE & $L_{\infty}$ \\
\hline DMM1 & 0.5 & $1.33 E-06$ & $4.13 E-07$ & $5.15 E-07$ \\
DMM2 & 0.5 & $1.62 E-06$ & $5.02 E-07$ & $7.09 E-07$ \\
[8] & 0.5 & $1.24 E-05$ & $3.24 E-06$ & - \\
{$[19]$} & 0.5 & - & $7.82 E-06$ & $3.11 E-05$ \\
[20] & 0.5 & $1.74 E-05$ & $7.15 E-05$ & $4.59 E-05$ \\
DMM1 & 1 & $6.64 E-07$ & $1.27 E-07$ & $2.12 E-07$ \\
DMM2 & 1 & $9.38 E-06$ & $1.79 E-06$ & $2.66 E-06$ \\
[8] & 1 & $2.67 E-05$ & $4.27 E-06$ & - \\
[9] & 1 & $9.97 E-04$ & $5.98 E-03$ & $2.27 E-03$ \\
{$[10]$} & 1 & $3.75 E-06$ & $2.47 E-05$ & $4.57 E-06$ \\
{$[19]$} & 1 & - & $2.07 E-05$ & $1.01 E-05$ \\
[20] & 1 & $8.15 E-06$ & $5.45 E-05$ & $2.51 E-05$ \\
DMM1 & 2 & $4.24 E-06$ & $6.24 E-07$ & $9.16 E-07$ \\
DMM2 & 2 & $2.76 E-06$ & $4.06 E-07$ & $5.30 E-07$ \\
{$[8]$} & 2 & $3.20 E-05$ & $3.94 E-06$ & - \\
{$[9]$} & 2 & $1.09 E-03$ & $8.50 E-03$ & $2.87 E-03$ \\
{$[10]$} & 2 & $4.47 E-06$ & $3.83 E-05$ & $5.61 E-06$ \\
{$[19]$} & 2 & - & $3.01 E-06$ & $8.25 E-06$ \\
{$[20]$} & 2 & $9.36 E-06$ & $8.13 E-05$ & $2.41 E-05$ \\
\hline
\end{tabular}

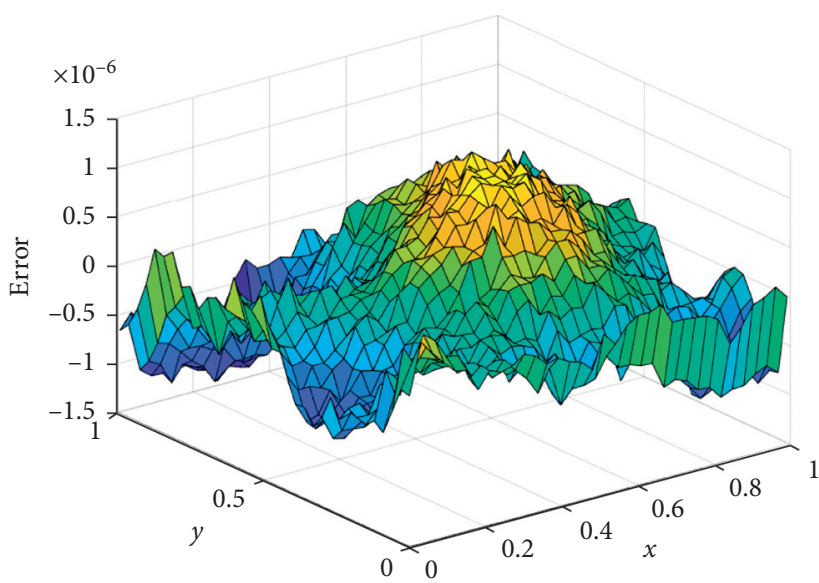

Figure 2: The absolute error for Example 1.

$$
\begin{gathered}
u(x, y, 0)=\exp (x+y), \quad(x, y) \in[0,1] \times[0,1], \\
u_{t}(x, y, 0)=-\exp (x+y), \quad(x, y) \in[0,1] \times[0,1],
\end{gathered}
$$

and the mixed boundary conditions

$$
\begin{gathered}
u(0, y, t)=\exp (y-t), \quad 0 \leq t<1, \\
u(1, y, t)=\exp (1+y-t), \quad 0 \leq t<1, \\
u(x, 1, t)=\exp (x+1-t), \quad 0 \leq t<1, \\
\frac{\partial u}{\partial t}(x, 0, t)=\exp (x-t), \quad 0 \leq t<1 .
\end{gathered}
$$

The corresponding analytical/exact solution is

$$
u(x, y, t)=\exp (x+y-t)
$$

with source term 


$$
f(x, y, t)=-2 \exp (x+y-t) .
$$

The coefficients are $\alpha=1, \beta=1$, and $\delta=1$.

Similar to the previous example, the root mean square errors (RMSE), $L_{2}$, and maximum errors, $L_{\infty}$, are shown in Table 2 for space and time step $h_{x}=h_{y}=\Delta t=(1 / 9)$. We compare our results with the reported results in $[9,10,19]$. The DMM time step $\Delta t=(1 / 9)$ is still larger than the one $\mathrm{d} t=0.001$ in $[9,10,19]$. As can be seen from the table, the numerical results are in good agreement with the exact solution. We can see that the results obtained by the proposed method are still consistent with the exact solutions and the maximum absolute errors remain stable around $L_{\infty} \approx 10^{-5}$ for all times tested. It is shown that the maximum absolute errors are a little larger than the previous example. This phenomenon may be partially due to the mixed boundary conditions.

5.3. Example 3. Here, we consider the hyperbolic telegraph equation of the form equation (1) in the interval $0 \leq x \leq 1$ with the analytical/exact solution:

$$
u(x, y, t)=\ln (1+x+y+t) .
$$

The corresponding source function and initial conditions are

$$
\begin{gathered}
f(x, t)=\frac{1}{1+x+y+t}+\ln (1+x+y+t)+\frac{1}{(1+x+y+t)^{2}}, \\
u(x, y, 0)=\ln (1+x+y), u_{t}(x, y, 0)=\frac{1}{1+x+y}, \quad 0 \leq x \leq 1,
\end{gathered}
$$

respectively. The boundary conditions are

$$
\begin{array}{ll}
u(0, y, t)=\ln (1+y+t), & 0 \leq t<1, \\
u(1, y, t)=\ln (2+y+t), & 0 \leq t<1, \\
u(x, 1, t)=\ln (x+2+t), & 0 \leq t<1, \\
u(x, 0, t)=\ln (1+x+t), & 0 \leq t<1 .
\end{array}
$$

For $h_{x}=h_{y}=\Delta t=(1 / 9)$, the total collocation point number is $N=1036$. Table 3 contains a comparison of the root mean square errors (RMSE), $L_{2}$, and maximum errors, $L_{\infty}$, for DMM to those obtained by the other numerical methods $[9,10,19]$ for different times $t=0.5, t=1, t=2$, $t=3$, and $t=5$. As can be seen from the table, the numerical results are in good agreement with the exact solutions. Our DMM results are more accurate than the other numerical methods.

5.4. Example 4. Let us consider the hyperbolic telegraph equation of the form equation (1) in the interval $0 \leq x \leq 1$ with the analytical/exact solution:

$$
u(x, y, t)=\exp (-t) \sinh (x)) \sinh (y) .
$$

The corresponding source function and initial conditions are
TABLE 2: Numerical results for Example 2.

\begin{tabular}{lcccc}
\hline Methods & $t$ & $L_{2}$ & RMSE & $L_{\infty}$ \\
\hline DMM1 & 0.5 & $6.08 E-06$ & $8.62 E-06$ & $1.85 E-05$ \\
[9] & 0.5 & $3.48 E-03$ & $8.42 E-05$ & $9.51 E-03$ \\
{$[10]$} & 0.5 & $1.18 E-04$ & - & $2.36 E-04$ \\
{$[19]$} & 0.5 & - & $7.10 E-04$ & $1.16 E-03$ \\
DMM1 & 1 & $7.60 E-06$ & $6.53 E-06$ & $9.94 E-06$ \\
{$[9]$} & 1 & $3.24 E-03$ & $1.29 E-04$ & $7.47 E-03$ \\
{$[10]$} & 1 & $1.04 E-04$ & - & $1.78 E-04$ \\
{$[19]$} & 1 & - & $4.36 E-04$ & $9.25 E-04$ \\
DMM1 & 2 & $1.15 E-04$ & $3.63 E-05$ & $8.56 E-05$ \\
{$[9]$} & 2 & $2.85 E-04$ & $3.10 E-05$ & $1.04 E-03$ \\
{$[10]$} & 2 & $9.01 E-06$ & - & $2.39 E-05$ \\
{$[19]$} & 2 & - & $7.23 E-05$ & $2.75 E-04$ \\
\hline
\end{tabular}

TABle 3: Numerical results for Example 3.

\begin{tabular}{lcccc}
\hline Methods & $t$ & $L_{2}$ & RMSE & $L_{\infty}$ \\
\hline DMM1 & 0.5 & $7.06 E-06$ & $2.67 E-06$ & $4.41 E-06$ \\
[9] & 0.5 & $1.07 E-03$ & $1.11 E-03$ & $2.47 E-03$ \\
[10] & 0.5 & $4.13 E-05$ & $4.50 E-05$ & $8.18 E-05$ \\
[19] & 0.5 & - & $1.13 E-05$ & $3.87 E-05$ \\
DMM1 & 1 & $1.45 E-05$ & $6.06 E-06$ & $1.06 E-05$ \\
[9] & 1 & $1.53 E-03$ & $1.33 E-03$ & $3.31 E-03$ \\
{$[10]$} & 1 & $6.38 E-05$ & $5.81 E-05$ & $9.35 E-05$ \\
[19] & 1 & - & $1.76 E-05$ & $2.55 E-05$ \\
DMM1 & 2 & $8.80 E-05$ & $4.27 E-05$ & $9.34 E-05$ \\
[9] & 2 & $4.65 E-04$ & $3.20 E-04$ & $1.14 E-03$ \\
{$[10]$} & 2 & $2.61 E-05$ & $1.89 E-05$ & $4.24 E-05$ \\
{$[19]$} & 2 & - & $8.93 E-06$ & $1.98 E-05$ \\
DMM1 & 3 & $2.08 E-05$ & $1.13 E-05$ & $2.34 E-05$ \\
[9] & 3 & $2.20 E-04$ & $1.30 E-04$ & $4.36 E-04$ \\
{$[10]$} & 3 & $1.06 E-05$ & $6.58 E-06$ & $1.79 E-05$ \\
{$[19]$} & 3 & - & $1.14 E-05$ & $2.09 E-05$ \\
DMM1 & 5 & $5.51 E-05$ & $3.45 E-05$ & $5.78 E-05$ \\
{$[9]$} & 5 & $1.72 E-04$ & $8.42 E-05$ & $3.48 E-04$ \\
{$[10]$} & 5 & $7.10 E-06$ & $3.65 E-06$ & $1.08 E-05$ \\
{$[19]$} & 5 & - & $1.42 E-05$ & $2.55 E-05$ \\
\hline
\end{tabular}

$$
\begin{aligned}
f(x, t) & =\left(-2 \alpha+\beta^{2}-1\right) \exp (-t) \sinh (x) \sinh (y), \\
u(x, y, 0) & =\sinh (x) \sinh (y), u_{t}(x, y, 0)=-\sinh (x) \sinh (y),
\end{aligned}
$$

respectively. The boundary conditions are

$$
\begin{array}{ll}
u(0, y, t)=0=u(x, 0, t), \quad 0 \leq t<1, & \\
u(1, y, t)=\exp (-t) \sinh (1) \sinh (y), & 0 \leq t<1, \\
u(x, 1, t)=\exp (-t) \sinh (x) \sinh (1), & 0 \leq t<1 .
\end{array}
$$

The numerical results are obtained using space step $h_{x}=$ $h_{y}=(1 / 9)$ and time step $\Delta t=(1 / 9)$. Tables 4 and 5 present the root mean square errors (RMSE), $L_{2}$, and maximum errors, $L_{\infty}$, for $\alpha=10, \beta=5$ and $\alpha=10, \beta=0$, respectively. We compare our results with the reported results in $[8,9,20]$. From these tables, we can see that numerical results of the DMM for times $t=0.5$ and $t=1$ are better than the other numerical methods. For time $t=2$, the DMM results are similar with the other numerical methods. 
TABLe 4: Numerical results with $\alpha=10$ and $\beta=5$ for Example 4.

\begin{tabular}{lcccc}
\hline Methods & $t$ & $L_{2}$ & RMSE & $L_{\infty}$ \\
\hline DMM1 & 0.5 & $1.76 E-06$ & $4.44 E-07$ & $9.47 E-07$ \\
[8] & 0.5 & $1.12 E-04$ & $3.30 E-05$ & - \\
[9] & 0.5 & $1.07 E-04$ & $1.11 E-04$ & $2.47 E-04$ \\
[20] & 0.5 & $1.14 E-04$ & $3.01 E-05$ & $7.13 E-05$ \\
DMM1 & 1 & $2.86 E-05$ & $4.38 E-06$ & $8.69 E-06$ \\
[8] & 1 & $1.81 E-04$ & $3.23 E-05$ & - \\
[9] & 1 & $1.53 E-04$ & $1.33 E-04$ & $3.31 E-04$ \\
[10] & 1 & $2.88 E-06$ & $1.80 E-05$ & $3.73 E-06$ \\
DMM1 & 2 & $3.15 E-04$ & $1.77 E-05$ & $3.21 E-05$ \\
[8] & 2 & $4.73 E-04$ & $3.12 E-05$ & - \\
[9] & 2 & $4.65 E-05$ & $3.20 E-04$ & $1.14 E-05$ \\
[20] & 2 & $1.93 E-04$ & $1.20 E-05$ & $2.51 E-05$ \\
\hline & & & &
\end{tabular}

TABle 5: Numerical results with $\alpha=10$ and $\beta=0$ for Example 4.

\begin{tabular}{lcccc}
\hline Methods & $t$ & $L_{2}$ & RMSE & $L_{\infty}$ \\
\hline DMM1 & 0.5 & $3.48 E-6$ & $8.80 E-7$ & $1.44 E-6$ \\
[8] & 0.5 & $1.12 E-4$ & $3.31 E-5$ & - \\
[9] & 0.5 & $9.30 E-5$ & $3.47 E-4$ & $4.23 E-4$ \\
[20] & 0.5 & $1.53 E-4$ & $3.91 E-5$ & $9.52 E-5$ \\
DMM1 & 1 & $1.14 E-05$ & $1.75 E-06$ & $3.53 E-06$ \\
[8] & 1 & $1.86 E-04$ & $3.34 E-05$ & - \\
[9] & 1 & $6.37 E-04$ & $3.91 E-04$ & $2.58 E-04$ \\
[20] & 1 & $2.80 E-04$ & $4.35 E-05$ & $9.74 E-05$ \\
DMM1 & 2 & $3.29 E-04$ & $1.86 E-05$ & $3.44 E-05$ \\
[8] & 2 & $5.18 E-04$ & $3.41 E-05$ & - \\
[9] & 2 & $2.55 E-05$ & $4.27 E-04$ & $9.58 E-05$ \\
[20] & 2 & $5.38 E-04$ & $3.07 E-05$ & $8.45 E-05$ \\
\hline
\end{tabular}

5.5. Example 5. Consider the hyperbolic telegraph equation of the form equation (1) in the interval $0 \leq x \leq 1$ with the analytical/exact solution:

$$
u(x, y, t)=\cos (t) \sinh (x) \sinh (y) .
$$

The corresponding source function and initial conditions are

$$
\begin{aligned}
f(x, t) & =\left(-3 \cos (t)-2 \alpha \sin (t)+\beta^{2} \cos (t)\right) \sinh (x) \sinh (y), \\
u(x, y, 0) & =\sinh (x) \sinh (y), u_{t}(x, y, 0)=0,
\end{aligned}
$$

respectively. The boundary conditions are

$$
\begin{aligned}
& u(0, y, t)=0, u(x, 0, t)=\cos (t) \sinh (x), \quad 0 \leq t<1, \\
& u(1, y, t)=\cos (t) \sinh (1) \sinh (y), \quad 0 \leq t<1, \\
& u(x, 1, t)=\cos (t) \sinh (x) \sinh (1), \quad 0 \leq t<1 .
\end{aligned}
$$

The numerical results are obtained using space step $h_{x}=$ $h_{y}=(1 / 9)$ and time step $\Delta t=(1 / 9)$. Tables 6 and 7 show the root mean square errors (RMSE), $L_{2}$, and maximum errors, $L_{\mathrm{\infty}}$, for $\alpha=50, \beta=5$ and $\alpha=10, \beta=5$, respectively. We compare our results with the reported results in [8-10]. As can be seen from these tables, numerical results for times
TABLE 6: Numerical results with $\alpha=50$ and $\beta=5$ for Example 5.

\begin{tabular}{lcccc}
\hline Methods & $t$ & $L_{2}$ & RMSE & $L_{\infty}$ \\
\hline DMM1 & $t=0.5$ & $1.27 E-06$ & $4.65 E-07$ & $9.54 E-07$ \\
[8] & $t=0.5$ & $2.87 E-05$ & $1.23 E-05$ & - \\
[9] & $t=0.5$ & $9.88 E-05$ & $1.27 E-05$ & $3.70 E-04$ \\
[10] & $t=0.5$ & $1.83 E-06$ & $4.94 E-06$ & $2.55 E-06$ \\
DMM1 & $t=1$ & $7.77 E-06$ & $1.75 E-06$ & $4.54 E-06$ \\
[8] & $t=1$ & $7.01 E-05$ & $1.84 E-05$ & - \\
[9] & $t=1$ & $1.68 E-04$ & $3.51 E-05$ & $5.69 E-04$ \\
[10] & $t=1$ & $2.85 E-06$ & $1.25 E-05$ & $3.89 E-06$ \\
DMM1 & $t=2$ & $3.25 E-05$ & $5.63 E-06$ & $9.56 E-06$ \\
[8] & $t=2$ & $6.78 E-05$ & $1.37 E-05$ & - \\
[9] & $t=2$ & $1.71 E-04$ & $4.64 E-05$ & $5.26 E-04$ \\
[10] & $t=2$ & $2.28 E-06$ & $1.30 E-05$ & $3.03 E-06$ \\
DMM1 & $t=3$ & $7.90 E-06$ & $5.82 E-06$ & $8.13 E-06$ \\
[8] & $t=3$ & $1.39 E-05$ & $6.68 E-06$ & - \\
[9] & $t=3$ & $1.74 E-05$ & $1.99 E-06$ & $4.35 E-05$ \\
[10] & $t=3$ & $1.56 E-06$ & $2.53 E-06$ & $1.75 E-06$ \\
\hline
\end{tabular}

Table 7: Numerical results with $\alpha=10$ and $\beta=5$ for Example 5.

\begin{tabular}{lcccc}
\hline Methods & $t$ & $L_{2}$ & RMSE & $L_{\infty}$ \\
\hline DMM1 & $t=0.5$ & $2.58 E-06$ & $9.42 E-E-07$ & $1.87 E-06$ \\
[8] & $t=0.5$ & $2.01 E-05$ & $8.61 E-06$ & - \\
[9] & $t=0.5$ & $1.07 E-04$ & $1.38 E-05$ & $3.76 E-04$ \\
[10] & $t=0.5$ & $1.40 E-06$ & $3.80 E-06$ & $1.87 E-06$ \\
DMM1 & $t=1$ & $3.48 E-06$ & $7.83 E-07$ & $2.03 E-06$ \\
[8] & $t=1$ & $4.60 E-05$ & $1.21 E-05$ & - \\
[9] & $t=1$ & $1.72 E-04$ & $3.60 E-05$ & $5.64 E-04$ \\
[10] & $t=1$ & $1.99 E-06$ & $8.78 E-06$ & $2.59 E-06$ \\
DMM1 & $t=2$ & $2.12 E-05$ & $3.67 E-06$ & $6.08 E-06$ \\
[8] & $t=2$ & $4.45 E-05$ & $9.01 E-06$ & - \\
[9] & $t=2$ & $1.65 E-04$ & $4.47 E-05$ & $5.13 E-04$ \\
{$[10]$} & $t=2 a t=2$ & $1.42 E-06$ & $8.11 E-06$ & $1.83 E-06$ \\
DMM1 & $t=3$ & $1.49 E-05$ & $6.00 E-06$ & $7.71 E-06$ \\
[8] & $t=3$ & $6.48 E-06$ & $3.13 E-06$ & - \\
[9] & $t=3$ & $8.99 E-06$ & $1.03 E-06$ & $1.96 E-05$ \\
{$[10]$} & $t=3$ & $5.44 E-07$ & $1.30 E-06$ & $7.64 E-07$ \\
\hline
\end{tabular}

$t=0.5$ and $t=1$ are better than the other numerical methods. For times $t=2$ and $t=3$, the DMM results are similar with the other numerical methods.

\section{Conclusions}

A new direct meshless scheme is presented for the twodimensional second-order hyperbolic telegraph equations. The present numerical procedure considered two schemes for the basis functions from radial and nonradial aspects. There is no need to remove time-dependent variable during the whole solution process. The time-dependent variable is treated regularly during the whole solution process, and the hyperbolic telegraph equations can be solved in a direct way. From the numerical results, we can conclude that the proposed meshless method is superior to the other numerical methods. Besides, the direct meshless method can be extended to solve nonlinear problems with the Newton iterative method considered and high-order differential 
equations [39-41]. The DMM with the localized method or the domain decomposition method is promising in developing algorithms for large-scale problems.

\section{Data Availability}

The data used to support the findings of this study are available from the corresponding author upon request.

\section{Conflicts of Interest}

The authors declare that there are no conflicts of interest regarding the publication of this paper.

\section{Acknowledgments}

The work was supported by the Natural Science Foundation of Anhui Province (project no. 1908085QA09) and University Natural Science Research Project of Anhui Province (project nos. KJ2019A0591 and KJ2020B06).

\section{References}

[1] R. G. Cheng and H. X. Ge, "Element-free Galerkin (EFG) method for a kind of two-dimensional linear hyperbolic equation," Chinese Physics B, vol. 18, pp. 4059-4064, 2009.

[2] I. Ahmad, H. Ahmad, A.E. Abouelregal, P. Thounthong, and M. Abdel-Aty, "Numerical study of integer-order hyperbolic telegraph model arising in physical and related sciences," European Physical Journal-Plus, vol. 135, p. 759, 2020.

[3] R. K. Mohanty and M. K. Jain, "An unconditionally stable alternating direction implicit scheme for the two space dimensional linear hyperbolic equation," Numerical Methods for Partial Differential Equations, vol. 17, no. 6, pp. 684-688, 2001.

[4] R. K. Mohanty, "An operator splitting method for an unconditionally stable difference scheme for a linear hyperbolic equation with variable coefficients in two space dimensions," Applied Mathematics and Computation, vol. 152, no. 3, pp. 799-806, 2004.

[5] R. K. Mohanty, "New unconditionally stable difference schemes for the solution of multi-dimensional telegraphic equations," International Journal of Computer Mathematics, vol. 86, no. 12, pp. 2061-2071, 2009.

[6] B. Bülbül and M. Sezer, "A Taylor matrix method for the solution of a two-dimensional linear hyperbolic equation," Applied Mathematics Letters, vol. 24, no. 10, pp. 1716-1720, 2011.

[7] M. Dehghan, S. A. Yousefi, and A. Lotfi, "The use of He's variational iteration method for solving the telegraph and fractional telegraph equations," International Journal for Numerical Methods in Biomedical Engineering, vol. 27, no. 2, pp. 219-231, 2011.

[8] R. Jiwari, S. Pandit, and R. C. Mittal, "A differential quadrature algorithm to solve the two dimensional linear hyperbolic telegraph equation with Dirichlet and Neumann boundary conditions," Applied Mathematics and Computation, vol. 218, no. 13, pp. 7279-7294, 2012.

[9] R. C. Mittal and R. Bhatia, "A numerical study of two dimensional hyperbolic telegraph equation by modified B-spline differential quadrature method," Applied Mathematics and Computation, vol. 244, pp. 976-997, 2014.
[10] B. K. Singh and P. Kumar, "An algorithm based on a new DQM with modified extended cubic B-splines for numerical study of two dimensional hyperbolic telegraph equation," Alexandria Engineering Journal, vol. 57, no. 1, pp. 175-191, 2018.

[11] R. M. Hafez, "Numerical solution of linear and nonlinear hyperbolic telegraph type equations with variable coefficients using shifted Jacobi collocation method," Computational and Applied Mathematics, vol. 37, no. 4, pp. 5253-5273, 2018.

[12] H. Zheng, F. Wang, C. S. Chen, L. Min, and Y. Wang, "Improved 3D surface reconstruction via the method of fundamental solutions," Numerical Mathematics: Theory, Methods and Applications, vol. 13, no. 4, pp. 973-985, 2020.

[13] H. Zheng, C. Zhou, D.-J. Yan, Y.-S. Wang, and C. Zhang, "A meshless collocation method for band structure simulation of nanoscale phononic crystals based on nonlocal elasticity theory," Journal of Computational Physics, vol. 408, p. 109268, 2020.

[14] H. Zheng, C. Zhang, and Z. Yang, "A local radial basis function collocation method for band structure computation of 3D phononic crystals," Applied Mathematical Modelling, vol. 77, pp. 1954-1964, 2020.

[15] M. Dehghan and A. Mohebbi, "High order implicit collocation method for the solution of two-dimensional linear hyperbolic equation," Numerical Methods for Partial Differential Equations, vol. 25, no. 1, pp. 232-243, 2009.

[16] M. Dehghan and A. Shokri, "A meshless method for numerical solution of a linear hyperbolic equation with variable coefficients in two space dimensions," Numerical Methods for Partial Differential Equations, vol. 25, no. 2, pp. 494-506, 2009.

[17] M. Dehghan and A. Ghesmati, "Combination of meshless local weak and strong (MLWS) forms to solve the two dimensional hyperbolic telegraph equation," Engineering Analysis with Boundary Elements, vol. 34, no. 4, pp. 324-336, 2010.

[18] M. Dehghan and R. Salehi, "A method based on meshless approach for the numerical solution of the two-space dimensional hyperbolic telegraph equation," Mathematical Methods in the Applied Sciences, vol. 35, no. 10, pp. 1220-1233, 2012.

[19] S. Abbasbandy, H. Roohani Ghehsareh, I. Hashim, and A. Alsaedi, "A comparison study of meshfree techniques for solving the two-dimensional linear hyperbolic telegraph equation," Engineering Analysis with Boundary Elements, vol. 47, pp. 10-20, 2014.

[20] D. Rostamy, M. Emamjome, and S. Abbasbandy, "A meshless technique based on the pseudospectral radial basis functions method for solving the two-dimensional hyperbolic telegraph equation," European Physical Journal-Plus, vol. 132, p. 263, 2017.

[21] J. Lin, F. Chen, Y. Zhang, and J. Lu, "An accurate meshless collocation technique for solving two-dimensional hyperbolic telegraph equations in arbitrary domains," Engineering Analysis with Boundary Elements, vol. 108, pp. 372-384, 2019.

[22] M. Aslefallah and D. Rostamy, "Application of the singular boundary method to the two-dimensional telegraph equation on arbitrary domains," Journal of Engineering Mathematics, vol. 118, no. 1, pp. 1-14, 2019.

[23] Y. Zhou, W. Qu, Y. Gu, and H. Gao, "A hybrid meshless method for the solution of the second order hyperbolic telegraph equation in two space dimensions," Engineering Analysis with Boundary Elements, vol. 115, pp. 21-27, 2020. 
[24] S. Reutskiy, Y. Zhang, J. Lin, and H. Sun, "Novel numerical method based on cubic B-splines for a class of nonlinear generalized telegraph equations in irregular domains," Alexandria Engineering Journal, vol. 59, no. 1, pp. 77-90, 2020.

[25] E. V. Glushkov, N. V. Glushkova, and C. S. Chen, "SemiAnalytical solution to heat transfer problems using Fourier transform technique, radial basis functions, and the method of fundamental solutions," Numerical Heat Transfer, Part B: Fundamentals, vol. 52, no. 5, pp. 409-427, 2007.

[26] Y. Gu, X. He, W. Chen, and C. Zhang, "Analysis of threedimensional anisotropic heat conduction problems on thin domains using an advanced boundary element method," Computers \& Mathematics with Applications, vol. 75, no. 1, pp. 33-44, 2018.

[27] R. M. Cotta, C. P. Naveira-Cotta, D. C. Knupp, J. L. Z. Zotin, P. C. Pontes, and A. P. Almeida, "Recent advances in computational-analytical integral transforms for convection-diffusion problems," Heat and Mass Transfer, vol. 54, no. 8, pp. 2475-2496, 2018.

[28] J. Ravnik and J. Tibuat, "Fast boundary-domain integral method for unsteady convection-diffusion equation with variable diffusivity using the modified Helmholtz fundamental solution," Numerical Algorithms, vol. 82, no. 4, pp. 1441-1466, 2019.

[29] E. J. Kansa, "Multiquadrics-A scattered data approximation scheme with applications to computational fluid-dynamics-II solutions to parabolic, hyperbolic and elliptic partial differential equations," Computers \& Mathematics with Applications, vol. 19, no. 8-9, pp. 147-161, 1990.

[30] B. Fornberg, E. Larsson, and N. Flyer, "Stable computations with Gaussian radial basis functions," SIAM Journal on Scientific Computing, vol. 33, no. 2, pp. 869-892, 2011.

[31] D. E. Myers, "Anisotropic radial basis functions," International Journal of Pure and Applied Mathematics, vol. 42, pp. 197-203, 2008.

[32] D. E. Myers, S. De Iaco, D. Posa, and L. De Cesare, "Spacetime radial basis functions," Computers \& Mathematics with Applications, vol. 43, no. 3-5, pp. 539-549, 2002.

[33] K. Parand and J. A. Rad, "Kansa method for the solution of a parabolic equation with an unknown spacewise-dependent coefficient subject to an extra measurement," Computer Physics Communications, vol. 184, no. 3, pp. 582-595, 2013.

[34] C.-Y. Ku, C.-Y. Liu, J.-E. Xiao, and M.-R. Chen, "Solving backward heat conduction problems using a novel space-time radial polynomial basis function collocation method," Applied Sciences, vol. 10, no. 9, p. 3215, 2020.

[35] F. Wang, W. Chen, and X. Jiang, "Investigation of regularized techniques for boundary knot method," International Journal for Numerical Methods in Biomedical Engineering, vol. 26, no. 12, pp. 1868-1877, 2010.

[36] F. Wang, W. Chen, and L. Ling, "Combinations of the method of fundamental solutions for general inverse source identification problems," Applied Mathematics and Computation, vol. 219, no. 3, pp. 1173-1182, 2012.

[37] G. E. Fasshauer and J. G. Zhang, "On choosing "optimal" shape parameters for RBF approximation," Numerical Algorithms, vol. 45, no. 1-4, pp. 345-368, 2007.

[38] W. Chen, Y. Hong, and J. Lin, "The sample solution approach for determination of the optimal shape parameter in the Multiquadric function of the Kansa method," Computers \& Mathematics with Applications, vol. 75, no. 8, pp. 2942-2954, 2018.

[39] O. Bazighifan and H. Ramos, "On the asymptotic and oscillatory behavior of the solutions of a class of higher-order differential equations with middle term," Applied Mathematics Letters, vol. 107, Article ID 106431, 2020.

[40] O. Bazighifan, "Kamenev and Philos-types oscillation criteria for fourth-order neutral differential equations," Advances in DifferenceEquations.vol. 2020, p. 201, 2020.

[41] O. Bazighifan, "On the oscillation of certain fourth-order differential equations with p-Laplacian like operator," Applied Mathematics and Computation, vol. 386, Article ID 125475, 2020. 